\title{
Tensiones y ambivalencias durante el trabajo de cuidados. Estudio de caso de un Servicio de Ayuda a Domicilio en la provincia de Barcelona ${ }^{1}$
}

\author{
Mireia Roca Escoda ${ }^{2}$
}

Recibido: 17 de mayo de 2016 / Aceptado: 12 de octubre de 2016

Resumen. El cuidado de las personas mayores ha dejado de ser una cuestión exclusivamente privada para pasar a ser también una cuestión pública, traspasando así diversos ámbitos e implicando a diferentes agentes sociales. Mediante una metodología de investigación cualitativa, basada en un estudio de caso etnográfico, se han realizado entrevistas en profundidad a trabajadoras del cuidado remunerado (trabajadoras familiares) y observación participante durante su práctica profesional. En este artículo analizamos el modelo de cuidado a personas mayores ofertado desde un servicio público de atención a domicilio. Un trabajo que convive con formas tradicionales de atención (a cargo de la familia, principalmente por parte de las mujeres), y donde la profesionalización no está consolidada. En este contexto aparecen las tensiones y ambivalencias que se manifiestan durante el servicio de cuidado profesional y se plantea la necesidad de nuevos debates sobre la responsabilidad social del cuidado y las características propias de la actividad cuando esta es remunerada.

Palabras clave: envejecimiento; trabajo de cuidados; género; servicio de ayuda domicilio; políticas públicas.

\section{[en] Contradictions and ambiguities during care work. Case study of a Home- Care-Service in the province of Barcelona}
Abstract. The care of the elderly people has stopped being an exclusively private matter, becoming a public question crossing different fields and involving different social agents. Through a qualitative research methodology, based on an ethnographic case-study, in-depth interviews have been made to paid professionals working in the care of the elderly (nurses of a home-care-service). This same me- thodology guided the participatory observation made during the professional praxis. In this article we analyse the model of public home-care-services for the elderly. A work that coexists with traditional ways (households, mainly women looking after elder family members), and where professionalization is not consolidated. In this context contradictions and ambiguities arise and call for the need of new de- bates on the social responsibility of the care of the elderly, as well as regarding the own characteristics of the activity when it is remunerated.
Key words: ageing; care work; gender; Home-Care-Service; public policy.
Sumario: 1. Introducción. 2. La organización social del cuidado. 2.1. El Servicio de Ayuda a Domici- lio. 3. El trabajo de cuidados en un SAD. 4. Tensiones y ambivalencias durante la práctica profesional.

\footnotetext{
El artículo forma parte del programa de Doctorado de Antropología Social y Cultural de la Universidad Autónoma de Barcelona.

Universidad Autónoma de Barcelona

E-mail: mrocaescoda@gmail.com
}
2 Estudiante de doctorado 
4.1. La organización del servicio: entre el domicilio y la empresa. 4.2. La identidad profesional: entre lo vocacional y lo familiar. 4.3. El contenido del trabajo de cuidados. 5. Conclusiones. 6. Bibliografía

Cómo citar: Roca Escoda, M. (2017) "Tensiones y ambivalencias durante el trabajo de cuidados. Estudio de caso de un Servicio de Ayuda a Domicilio en la provincia de Barcelona". Cuadernos de Relaciones Laborales 35(2), 371-391.

\section{Introducción}

El objetivo de este artículo es analizar la prestación de cuidados a personas mayores desde el Servicio de Atención Domiciliaria (SAD). Este servicio, que crece con la aprobación de la Ley estatal 39/2006 de Promoción de la Autonomía y Atención a las Personas en situación de Dependencia, convive con la atención familiar y es ejercido por nuevas profesionales, las Trabajadoras Familiares ${ }^{3}$. Pretendemos reconstruir las tensiones y ambivalencias que se generan en la práctica cotidiana contrastando las representaciones sociales en torno al cuidado y las previsiones de las políticas públicas. Tal como intentaremos mostrar, las dimensiones de género, clase y etnia se hallan presentes en la práctica profesional y las condiciones de ejercerla.

El envejecimiento de la población en Cataluña, como en el resto de España y en los países occidentales aumenta (según datos de la OCDE en España la población de más de 80 años era del $5 \%$ en 2010 y se prevé que en el 2050 sea del 15\%). A la vez, se incrementan las necesidades de cuidado a las personas mayores en situación de fragilidad que necesitan un apoyo. El cuidado de esas personas ha sido tradicionalmente realizado por la familia (principalmente por la mujer) en el ámbito doméstico, como una actividad no remunerada, socialmente devaluada e invisibilizada (Carrasco, Borderías, Torns, 2011; Comas d'Argemir, 2012). En el momento actual, diversos cambios sociales protagonizados por el envejecimiento de la población (aumento de las crecientes necesidades de cuidado a las personas mayores), los cambios en las estructuras familiares (Brullet, 2010) y la participación masiva de la mujer, con responsabilidades familiares, en el mercado de trabajo (que supone la disminución de tiempo dedicado al cuidado familiar), han hecho emerger la necesidad social de reorganizar el cuidado: "¿quién cuida a las personas mayores?" Este interrogante abre nuevos debates en torno a la responsabilidad social del cuidado (entre la familia, el estado, el mercado y la comunidad) y al valor del trabajo de cuidado, a la vez que cuestiona el sistema que sustentaba la función clave del modelo familiar en una sociedad patriarcal, organizada sobre la base de un modelo de trabajo productivo (masculino) y reproductivo (femenino) (Carrasco, Borderías, Torns, 2011).

Esta situación, conocida como "crisis del cuidado" (Pérez Orozco, 2006; Mande11, 2010; Batthyány, Genta y Perrotta, 2014), plantea el reto de organizar los cuidados teniendo en cuenta la contribución equitativa de hombres y mujeres al bienestar de la sociedad y el derecho a decidir cómo ser cuidado. El cuidado de las personas mayores ha dejado de ser una cuestión exclusivamente privada para pasar a ser también una cuestión pública (Tronto, 2004; Williams, 2010; Esquivel, 2011; Batthyány,

Nos referimos a las TF en femenino por la alta feminización del sector. 
Genta y Perrotta, 2014), traspasando así diversos ámbitos e implicando a diferentes agentes sociales: el "cuidado social” (Daly y Lewis, 2000).

\section{La organización social del cuidado}

Con la promulgación de la Ley de la Dependencia en 2006, aparece en España el derecho subjetivo ${ }^{4}$ a recibir cuidado, a fin de garantizar la cobertura de las necesidades de cuidado a toda la población. Se promueven diversos servicios públicos y prestaciones económicas para dar respuesta a las necesidades, cubiertas hasta el momento, exclusivamente, por las familias. El desarrollo autonómico del Sistema Nacional de Atención a la Dependencia presenta contornos muy diferentes en cada Comunidad Autónoma, lo que muestra una variedad de concepciones de las políticas sociales (Martínez-Buján, 2011). Aun así, lo común en todas ellas es el papel protagonista de la familia. Esta política responde al modelo de bienestar caracterizado por políticas conservadoras, basado en la familia como responsable de las necesidades sociales y la promoción de servicios asistenciales y subsidiarios (Rodríguez Cabrero, 2011). Según datos del Sistema para la Autonomía y Atención a la dependencia, en 2012 el 44,46\% de las prestaciones corresponden a la prestación económica destinada a los cuidados familiares, mientras que el Servicio de Ayuda a Domicilio sólo alcanzaba al 13,05\%, la atención diurna al 6,87\% y la atención residencial al 13,10\%. El resto se distribuye entre los servicios de teleasistencia, los servicios preventivos o la prestación económica de asistente personal (Instituto de Mayores y Servicios Sociales, 2012) . $^{5}$

Desde el inicio, la Ley de la Dependencia estableció que priorizaría los servicios sociales sobre las prestaciones económicas, pero en la práctica ha sucedido lo contrario, delegando el cuidado a las familias y favoreciendo la compra del cuidado a través del servicio doméstico. Así en 2008, la fuente más común de atención es el cuidado no remunerado (realizado principalmente por la familia), con un 78,5\%. Las personas que reciben cuidado remunerado solo ocupa el 10,9\% (siendo el privado un 7,3\%). El 10,5\% restante recibe tanto cuidado remunerado como no remunerado (Rogero-García y Ahmed-Mohamed, 2014). El desarrollo de la ley coincide también con la crisis económica en un contexto español gobernado por políticas conservadoras. Desde finales de 2011 diversas reformas legales y estructurales basadas en la reducción del financiamiento público (Deusdad, Comas d'Argemir y Dziegielewski, 2016) han provocado retrasos en la aplicación de la ley y no ha favorecido la sostenibilidad del sistema dando lugar al debilitamiento de los derechos reconocidos en un inicio. Es así como se reducen servicios y las prestaciones monetarias se generalizan, favoreciendo a los proveedores privados en un contexto donde la oferta pública de servicios es insuficiente (Marbán Gallego, 2011; Vilà, 2013), siguiendo una lógica neoliberal, que prescribe la austeridad y la rentabilidad de la política social, legitimando los recortes en los servicios sociales para las personas mayores dependientes (Peterson, 2015).

Las Administraciones garantizan la atención a las personas con dependencia, que son evaluadas por los equipos de valoración del sistema de dependencia. En función del grado de dependencia, tienen derecho a recibir servicios o prestaciones económicas.

Estos datos corresponden a los porcentajes totales en España. Para ver las diferencias regionales se pueden consultar los datos anuales publicados por el IMSERSO o el estudio de Martínez-Buján (2011). 
El cuidado mercantilizado aumentó en los últimos años y se hizo más visible como fuente de empleo (tanto en el caso de las empleadas del hogar como en el de las trabajadoras de servicios profesionales). El trabajo de cuidados remunerado en servicios residenciales y atención diurna en 2009 en Cataluña incluía a 37.404 personas contratadas y según estimaciones en 2013 a 41.395, el 1,4\% de la población ocupada en Cataluña. El número de trabajadores del SAD serían 7.400 en 2012, según estimaciones el $0,25 \%$ de la ocupación total en Cataluña (Asociación Catalana de Recursos Asistenciales, 2014). Se da también una diferencia en el origen de las trabajadoras contratadas, siendo las extranjeras las que predominan en el sector doméstico como empleadas del hogar. Según datos de la Encuesta de Población Activa, en 2009 las trabajadoras extranjeras ocupadas en trabajos de cuidados profesionales es del 14,6\% y en el ámbito del servicio doméstico del 60,4\% (Martínez-Buján, 2011).

A la vez, la presencia del mercado en este sector crece, ya sea por la compra de servicios al mercado como por la gestión privada de los servicios públicos por parte de organizaciones mercantiles o sociales. En 2012 solo un 16,5\% de los centros residenciales y un $27,3 \%$ de los centros de día son ofertadas directamente con financiamiento público, el resto son prestadas por entidades sociales y mercantiles directamente o mediante concertaciones. En el caso del SAD, las entidades públicas que prestan el servicio directamente representan el 14,8\% y las privadas el 85,2\% (Asociación Catalana de Recursos Asistenciales, 2014). Este modelo privatizador, orientado a la obtención de beneficios, favorece el clientelismo y afecta la calidad de la prestación y las condiciones laborales (Pérez Orozco, 2006; Recio, 2010; Martínez-Buján, 2010).

La profesionalización del sector laboral del cuidado ha aumentado, pero se mantiene el cuidado familiar (Torns, 2008; Brullet, 2010, Comas d'Argemir, 2012, 2015; Recio et al., 2015). Al mismo tiempo, el cuidado no profesional en el ámbito del servicio doméstico ha sido una alternativa seguida por muchas familias. El empleo doméstico es cuidador principal en el 6,4\% de las situaciones y se estima que un $14,5 \%$ de los adultos mayores con dependencia utilizan este recurso, siendo las mujeres inmigrantes las principales empleadas (Martínez-Buján, 2011). Esto es debido a la expansión de las transferencias monetarias sobre los servicios, que ha comportado la contratación de una empleada de hogar como cuidadora principal, muchas de ellas extranjeras. La escasez de recursos para abordar el cuidado, el "déficit del cuidado", ha provocado una reprivatización de la reproducción comportando procesos migratorios para cubrir la demanda de los países más ricos, dando lugar a las llamadas "cadenas mundiales de afecto y asistencia" (Hochschild, 2008). Estos procesos transfieren el cuidado de unas mujeres a otras y suponen la externalización del problema basada en relaciones globales de poder de género (Pérez Orozco, 2006), lo que conlleva que el cuidado que se ofrece a los países receptores de esta población femenina implique una "triple discriminación" en base a la etnia, la clase y el género (Parella, 2003).

\subsection{El Servicio de Ayuda a Domicilio}

En Cataluña, los servicios públicos que atienden en su domicilio a las personas en situación de dependencia, muchas de ellas personas mayores, son los Servicios de Ayuda a Domicilio ${ }^{6}(\mathrm{SAD})$. Forman parte de los llamados "servicios de proximidad",

6 Prestación básica regulada por la Ley estatal 36/2006 de Promoción de las Autonomía Personal y Atención a las personas en situación de Dependencia y la ley catalana 12/2007 de Servicios Sociales. 
denominación dada al trabajo doméstico asalariado, relacionado con la precariedad y el trabajo invisible (Parella, 2003; Martínez-Buján, 2010; Recio, 2010). Con la creación de los SAD nacen nuevas profesiones vinculadas al cuidado: las Trabajadoras Familiares $^{7}$ (las TF en adelante). Una profesión desarrollada principalmente por mujeres (ACRA, 2014), la mayoría de ellas autóctonas, a diferencia de las empleadas en el servicio doméstico donde predominan las mujeres inmigrantes (Vega, 2009; Martínez-Buján, 2011). El SAD ha experimentado un crecimiento desde su creación debido al incremento de las necesidades de los servicios bajo la idea de "proximidad" (personales y familiares). El crecimiento de los SAD tiene que ver también con la preocupación a nivel europeo por aumentar las tasas de ocupación como una solución ante el desempleo producido por la crisis de principios de los años 90. En Europa, a partir de la elaboración del Informe Delors, recogido en el Libro Blanco sobre competitividad y empleo (1993), se crearon nuevos filones de ocupación, destinados al colectivo de mujeres adultas con dificultades de inserción laboral, con el objetivo de crear nuevos empleos y afrontar nuevas necesidades sociales. En 2004, el Libro Blanco de la atención a las personas en situación de dependencia (Instituto de Mayores y servicios Sociales, 2004) contemplaba la creación de nuevos empleos por el desarrollo de los programas de atención. Se dijo que en España se generarían en neto por lo menos 267.057 puestos de trabajo a jornada completa en el año 2010, lo cual ayudaría a aflorar empleos que en aquel momento se venían realizando de forma sumergida o irregular. Resultaron trabajos femeninos, no cualificados, en condiciones laborales precarias, con pocos requerimientos formativos y pocas posibilidades de mejora profesional (Otaegui, 2007; Recio et al., 2015).

Los modelos políticos de provisión de cuidado llevan implícitos una definición de cuidado y unas ideas en torno a quien lo proporciona y sus beneficiarios, otorgando diferentes valores a la tarea de cuidar y dando lugar a un modelo social de organización del cuidado particular. El SAD es un recurso público que fomenta el cuidado social y su profesionalización, pero se desarrolla en un contexto caracterizado por un sistema de bienestar mediterráneo, caracterizado por la responsabilidad individual y familiar del cuidado (Rodríguez Cabrero, 2011; Comas d'Argemir, 2012, 2015). Es un recurso que fomenta la permanencia de la persona en el domicilio, en su entorno familiar, dando continuidad al imaginario del cuidado familiar, llevado a cabo en la intimidad por las mujeres, y que además supone incrementar la responsabilidad del cuidado a las familias. Todo ello de acuerdo con el discurso actual, que presenta a la familia como el elemento "natural" de convivencia. Siguiendo a Lewis (2007), las políticas no contribuyen a garantizar la valoración del trabajo de cuidado, están basadas en la individualización y en la desventaja para las mujeres.

El SAD se desarrolla en una cultura de cuidado familiar (y femenino) lo que dificulta su profesionalización y valoración social (Martínez-Buján, 2010; Recio et al., 2015). Se desarrolla en el marco de unas políticas públicas y unas organizaciones de servicio que avalan un sistema social basado en la desigualdad social y de género: condiciones de trabajo precarias, servicios subsidiarios, familistas y escasos (Otae-

Titulación obtenida inicialmente mediante formación profesional para la ocupación impartidos por los ayuntamientos. Desde 2003 se reconoce la profesión dentro de la formación reglada con el título Técnico de Atención Sociosanitaria (Formación Profesional de grado medio). Actualmente, hay tres vías de formación: la formación profesional, los certificados de profesionalidad y acreditación de competencias (reconocimiento de las competencias profesionales adquiridas mediante experiencia laboral). 
gui, 2007; Razavi y Staab, 2010; Recio, 2010; Recio et al., 2015; Tobío, et al., 2010). A fin de dar respuesta a las desigualdades de género y al reconocimiento del cuidado como trabajo profesional, garantizando los derechos de las personas proveedoras y receptoras de cuidado, es necesario conceptualizar el cuidado como valor público, alejarnos de la responsabilidad individual, en tanto que todos y todas somos receptores de cuidado. Un escenario donde es necesario cuestionar la asociación del rol de cuidadora a la identidad femenina, convirtiendo el trabajo de cuidado en responsabilidad social y en una tarea no feminizada (Fraser, 1997; Tronto, 2004).

\section{El trabajo de cuidados en el Sad}

En este artículo presentamos una aproximación etnográfica al trabajo de cuidados en el marco de un servicio público: el Servicio de Ayuda a Domicilio (SAD). Aunque la mayor parte del cuidado sigue estando prestado por las familias (las mujeres) dentro de la esfera doméstica y reproductiva, actualmente también es un trabajo remunerado. Nuestra aproximación se sitúa en los estudios orientados al análisis del cuidado y las políticas públicas, con el reclamo de que las sociedades contemplen el cuidado como responsabilidad social (Daly y Lewis, 2000, Esquivel, 2011, Razavi, 2007, Williams, 2010). Se trata de una perspectiva clave ya que incluye las instituciones y servicios de mercado en la provisión del cuidado. También permite captar el cuidado desde diferentes ámbitos y sacarlo de su espacio tradicional, el doméstico y femenino por excelencia, dando lugar a transformaciones en las modalidades de cuidado así como en las expectativas sobre quién debe cuidar y cómo debe hacerlo (Del Valle, 2004). Para el análisis del cuidado en este contexto, nos referimos al concepto de cuidado social introducido por Daly y Lewis (2000), que incluye las actividades y las relaciones para la satisfacción, las necesidades físicas y emocionales, los marcos normativos, económicos y sociales donde se desarrolla. Un modelo de trabajo que se configura por la interacción de distintas variables: el valor social de la labor de cuidados por la sociedad, el tipo de políticas sociales que regulan los servicios y las formas de gestionarlas, y la cualificación profesional de este tipo de empleo (Recio, 2010).

El objetivo general fue conocer las dinámicas relacionales, barreras culturales y representaciones sociales entorno al trabajo de cuidado a las personas mayores en un SAD. El objetivo se concretó en identificar las tensiones y ambivalencias que se producen durante la práctica profesional entre los agentes implicados en la prestación del servicio; las representaciones sociales entorno al cuidado que dificultan la igualdad social y de género; y el modelo de política social que promueve una organización del cuidado particular.

Las ideas que orientaron el estudio fueron que la organización del SAD conlleva tensiones y ambivalencias en la realización de la actividad y en la delimitación de la tarea como trabajo remunerado, ya que las estructuras normativas condicionan la forma del cuidado, creando profesiones y recursos sociales que dificultan su abordaje, en una sociedad donde el cuidado está estrechamente vinculado a lo íntimo y familiar (y femenino). Precisamente el modelo social basado en dicotomías (productivo y reproductivo; masculino y femenino; público y privado) dificulta el análisis del cuidado en este contexto institucional, que traspasa fronteras y se sitúa en los intersticios (Waerness, 1987; Comas y Roca, 1996; Hochschild, 2008). 
Se parte de la idea de que el cuidado es una construcción social. La naturaleza del cuidado varía según si se realiza dentro de la familia o en una actividad remunerada; por tanto, las ideas en torno a ella y los límites pueden ser difusos. El trabajo de cuidados remunerado exige no sólo capacidades de tipo material y práctico, sino también de tipo relacional y emocional, que son básicas para poder realizar el trabajo. Precisamente muchas de las dificultades para la institucionalización y la mercantilización del cuidado tienen que ver con estos últimos factores, factores que entorpecen el traslado de la actividad de hacerse cargo de los otros desde los entornos íntimos y familiares a la esfera pública o institucional (Hochschild, 2008; Molinier, 2008; Zelizer, 2009). Al ser trabajos que presentan características semejantes al trabajo doméstico (suponen unas cualidades vinculadas al ámbito privado), las fronteras entre trabajo remunerado y no remunerado se difunden, se transforman, poniendo en cuestión la dicotomía público/privado y dificultando la valoración y catalogación de este trabajo (Del Valle, 2004; Martin Palomo, 2008; Esquivel, 2011).

La organización social del cuidado continúa basándose en la desigualdad social en base al género y la clase, siendo el trabajo de cuidados una profesión feminizada y poco valorada. Las representaciones sociales y las barreras de oportunidad sobre quién realiza el trabajo de cuidados se basan en patrones de género y clase, tanto por la vinculación del cuidado a la identidad femenina como por las condiciones laborales del sector (Torns, 2008; Carrasco, 2009). La feminización del cuidado se relaciona con la mercantilización de los procesos productivos fuera del ámbito doméstico y la vinculación de las mujeres a la domesticidad, lo que contribuye a naturalizar las responsabilidades del cuidado y a construir nuevas identidades femeninas asociadas al cuidado (Juliano, 1989). Tal y como dice Torns (2008), en estos trabajos femeninos, no se valora tanto el "saber hacer" o el "saber decir", sino el "saber estar". Es debido a la feminización del trabajo, que al pasar a la esfera pública, sigue estando poco valorada. Es un sector laboral precario porque se asocia al trabajo doméstico, esto es lo que explica la catalogación de estas profesiones como no especializadas y con bajos salarios (Borderías, 2000). En este sentido, diversos estudios feministas analizan cómo la aplicación de categorías de análisis del trabajo remunerado que responden al modelo masculino, interpretan el trabajo femenino como de segunda clase, poco cualificado y marginal, hecho que dificulta su valoración y su importancia en el funcionamiento económico y social (Himmelweit, 2000; Borderías, 2000; Razavi, 2007). La idea de que el cuidado requiere de unas cualidades que han sido feminizadas, habilidades y capacidades aprendidas en el entorno doméstico, concebida como una tarea vocacional, no aprendida y vinculada a la mujer (Juliano, 1989), también dificulta la entrada del hombre al trabajo de cuidado, tanto por los potenciales cuidadores como por los receptores y gestores de los cuidados. Pero la incorporación progresiva de los hombres en este ámbito, que puede estar relacionado con la mayor demanda de cuidado y la situación de desempleo de muchos hombres, pone en cuestión la vinculación de la mujer al cuidado y abre nuevos interrogantes en relación a la identidad de género y su carácter dicotómico ("femenino" - "masculino") partiendo de la idea de que el género es una construcción social (Butler, 1990; Stolcke, 2003).

Para llevar a cabo la investigación hemos utilizado la estrategia de caso etnográfico, basado en el trabajo de campo y las técnicas cualitativas; la relación entre la teoría y los datos empíricos y la visión crítica del fenómeno objeto de estudio. Se escogieron como unidades de análisis los discursos, prácticas y relaciones que se establecen en relación al cuidado a las personas mayores prestado por las TF, las que 
realizan la atención personal en los domicilios. A pesar de que los discursos de los gestores del servicio y los beneficiarios del cuidado se han tenido en cuenta, nos ha interesado especialmente la perspectiva de las profesionales de atención directa en relación a su trabajo.

La unidad de observación fue un SAD de Mataró (provincia de Barcelona). Dado que el ayuntamiento externaliza la producción del servicio a cuatro entidades privadas, se eligió para el estudio una empresa mercantil. El SAD del estudio está integrado por distintos profesionales: 1 jefe de servicio, 2 coordinadoras técnicas, 35 Trabajadoras Familiares (TF) dedicadas a la atención directa y 13 auxiliares del hogar $(\mathrm{AH})$, dedicadas a la limpieza del hogar. Del total de las TF, 34 son mujeres. La mayoría responden a un perfil de mujeres autóctonas (del total, 6 son inmigradas). Sus edades oscilan entre los 30 y los 60 años, y el mayor volumen está representado por las de 40-50 años. Tienen contratos indefinidos de obra y servicio y trabajan entre 5 y 37 horas semanales, aunque la mayoría están entre las 20 y las 37 horas. La antigüedad en la empresa es, en primer lugar, entre 1 y 3 años, seguido de entre 3 y 6 años. Generalmente tienen experiencia laboral en otros SAD, en residencias para gente mayor o en domicilios a nivel privado. En 2011, la empresa atiende a un total de 274 personas usuarias (en lenguaje de la Administración y de los servicios), 68 hombres y 206 mujeres, que son derivados desde los servicios sociales del ayuntamiento. Del total de personas usuarias, 189 reciben el servicio de atención y 160 reciben el servicio de limpieza del hogar-aunque también hay personas que reciben ambos-. En cuanto al perfil de personas usuarias, el 69\% son personas de más de 65 años y el resto tienen problemas de salud mental (21\%) o son casos de infancia en situación de riesgo (10\%). Las personas mayores normalmente tienen apoyo familiar, pero como es insuficiente, sus necesidades son, principalmente, ayuda en la realización de la higiene personal, en la organización del hogar, en la ejecución de la compra y acompañamiento en el paseo.

Se realizó una exploración bibliográfica sobre el tema de estudio y se procedió al análisis cuantitativo de fuentes estadísticas. Durante el trabajo de campo se realizaron entrevistas en profundidad a los distintos actores que participan en la organización, gestión y atención del SAD: profesionales de atención directa ( 8 mujeres y 1 hombre), a las coordinadoras técnicas y al jefe del servicio. También desarrollamos observación participante durante la prestación del servicio y observaciones durante el trabajo diario que desempeñan las TF en los distintos domicilios. El material empírico también proviene de la observación y la participación directa en la coordinación técnica de un SAD (distinto del SAD objeto de estudio pero que gestiona la misma empresa), de donde se han podido extraer datos utilizados para contrastar y completar las informaciones obtenidas. Una experiencia profesional que desde una mirada antropológica nos ayudó a problematizar el objeto de estudio y avanzar en el análisis. Fue fundamental mostrar la interrelación existente entre los comportamientos y eventos observados, aquellos registrados a partir del discurso oral de los informantes y los que derivan de la lectura de documentos y de materiales bibliográficos. 


\section{Tensiones y ambivalencias durante la práctica profesional}

\subsection{La organización del servicio: entre el domicilio y la empresa}

El SAD es un servicio público que inicialmente se gestionaba directamente desde los servicios sociales municipales. Con el crecimiento de la demanda, el servicio se externalizó parcialmente, delegando solo la contratación de personal para la realización del servicio a un operador externo privado, y manteniendo la gestión del servicio desde servicios municipales (algunas de las TF entrevistadas estaban contratadas desde 1993 por una entidad privada pero formaban parte de los equipos municipales de gestión del SAD). Desde 2002, de acuerdo con la tendencia general de las administraciones públicas, la gestión del servicio se externaliza más, es decir, la provisión del servicio corresponde a la administración local a través de los servicios sociales y la producción del servicio se deriva a un productor externo mediante un concurso público. La empresa que gestiona el SAD objeto de estudio es una multinacional de servicios que se dedica a varios sectores, entre los cuales está el de servicios sociales. Es una empresa mercantil orientada a la producción de capital económico, bajo los criterios de eficiencia, rentabilidad y competitividad. La gestión del servicio se desarrolla en dos fases: en primer lugar, los servicios sociales municipales identifican a las personas usuarias, asignan el recurso y desarrollan el seguimiento y la gestión del caso (la revisión periódica del diagnóstico que dio lugar a la asignación del servicio); en segundo lugar derivan la demanda de servicio a una empresa privada, encargada de prestar el servicio, definiendo un circuito de trabajo y unos protocolos de actuación que reflejan las actividades y tareas que se llevan a cabo por las distintas profesionales, manteniendo una coordinación y seguimiento con la entidad pública. La coordinación diaria del servicio está a cargo de la coordinadora técnica de la empresa, quien recibe las demandas desde servicios sociales y asigna a las personas usuarias las profesionales de atención directa. Según si el servicio es de atención o de limpieza se asigna la profesional, acorde con su perfil profesional y su disponibilidad. Se adjudican unas horas de atención al día o a la semana al usuario beneficiario del servicio en función de la situación socioeconómica y/o las necesidades derivadas de la situación de dependencia. Si tienen capacidad económica, pagaran una parte del servicio (copago). Es el único recurso de atención domiciliar por parte de un profesional contratado desde un ente público que se ha ido configurando como una prestación básica de apoyo, tanto para quienes necesitan ayuda en la vida diaria como para sus cuidadores. Al no existir una normativa específica del SAD, son los ayuntamientos los que establecen las condiciones del servicio mediante un pliego de cláusulas técnicas y administrativas, pero también es la oferta y la organización de la empresa privada la que configura la estructura del servicio.

Las condiciones laborales de las TF están regidas por el convenio del sector ${ }^{8}$, pero sobre todo dependen de cómo lo aplica la entidad o empresa que contrata (tipología de contrato, horarios, desplazamientos, dietas, formación, etc.). Todas las

En Cataluña se aplica el IV Convenio colectivo de empresas de atención domiciliaria de Cataluña o el VI Convenio Marco Estatal de Servicios de Atención a las Personas Dependientes y Desarrollo de la Promoción de la Autonomía Personal. 
TF manifiestan que el salario que obtienen es muy bajo ${ }^{9}$ y no se corresponde con la dureza y valor de su trabajo. El hecho de que los contratos estén sujetos a variaciones de horas en función de la asignación semanal de personas usuarias no les da una estabilidad laboral, y que los servicios puedan variar en las franjas horarias tampoco les da garantía de un horario estable. El ritmo de trabajo es intenso, puesto que de un domicilio a otro hay poco margen de tiempo, los desplazamientos están muy acotados y no se establecen pausas dentro de la jornada laboral. A causa de estas condiciones, y de que el tiempo destinado a cada domicilio es muy limitado, la programación encadenada no permite flexibilizar el servicio, aspecto que dificulta un trabajo que se caracteriza por no ser pautado. A menudo, las TF están más rato en cada domicilio que el que tienen asignado, invirtiendo un tiempo que no se contabiliza y que provoca que los domicilios se solapen. De esta forma, tanto la organización del servicio como la regulación del trabajo por parte de la empresa, no ofrecen unas buenas condiciones laborales para las trabajadoras, siendo estas condiciones precarias. Tanto las bajas remuneraciones como la difícil estabilidad del trabajo reflejan la dimensión de las desigualdades de clase en el SAD. Aunque las profesionales reconocen que socialmente no se valora su trabajo (expresan que es una actividad dura y mal remunerada), sí que se sienten reconocidas y valoradas por las personas usuarias, rasgo indispensable para poder desarrollar su labor. Es la satisfacción y la realización personal en el desarrollo de esta ocupación, lo que lleva a las TF a no cambiar de trabajo, tal y como comenta Rosario ${ }^{10}$ :

Un porcentaje del trabajo es satisfacción que te da a ti... y de decir, he hecho mi trabajo bien. Aunque vayas de bólido al siguiente servicio, te encuentras satisfecha porque has realizado bien tu trabajo. Es una satisfacción muy grande que pocos trabajos te la dan. Está muy mal pagado pero en pocos trabajos tienes esta satisfacción, y a parte cuando te hablan y te dicen "gracias, me has ayudado", a mí eso me llena mucho. (Rosario)

Las TF desarrollan su trabajo en el domicilio de la persona, pero el trabajo de cuidados está sujeto a diferentes normas, ideas y reglas de los diferentes agentes implicados en el SAD: las que derivan de la organización del servicio (los servicios sociales y la empresa), las del domicilio y las de la práctica profesional. Las tareas a realizar en el domicilio se establecen primero por encargo de los servicios sociales, que definen unos objetivos de trabajo, y después en el domicilio, cuando se negocian con las personas usuarias. Si bien las decisiones sobre los servicios son externas, donde se concretan es en el ámbito privado, según las normas del usuario y las normas profesionales en el desarrollo de las tareas:

No es como trabajar en una empresa, en donde cada día entras en el mismo lugar, o trabajar en una residencia, en que siempre sigues las mismas normas, las de la institución. Aquí las normas son el domicilio. Hay las normas técnicas que no se

El IV Convenio colectivo de empresas de atención domiciliaria de Cataluña (2015) establece un salario mensual de 1066,16 € para la categoría de Trabajador Familiar; y un salario mensual de 914,12 € para la categoría de Auxiliar de Limpieza.

10 Las entrevistas realizadas a Ángeles, Teresa, Susana y María originalmente son en catalán, la traducción es nuestra. 
negocian, como hacer la higiene, por ejemplo de arriba abajo, como curar una úlcera, y las normas de cada casa, cómo quieren las toallas dobladas, qué orden de las cosas, etc., que estas las fija cada usuario, y te has de adaptar. Estamos en su terreno, es su vida íntima, has de ser neutro, no traspasar la barrera. (Ángeles)

Las personas receptoras de cuidados se muestran más o menos receptivas a recibir atención personal, pero las TF manifiestan que desarrollar su trabajo de entrada no es fácil. El hecho de entrar en el domicilio privado comporta que haya un proceso de adaptación y negociación de las dos partes, tanto a nivel de formas de hacer como de lo que se debe hacer (las necesidades pueden ser distintas según la percepción del usuario, de la TF y lo que establecen los servicios sociales). Así, se convierten en intermediarias entre el domicilio y la empresa; son quienes acaban detectando necesidades y estableciendo nuevas tareas, paralelamente a los servicios sociales o a la empresa, con todas las connotaciones que implica un trabajo individual y solitario. Lo ejemplifica muy bien una TF:

Sí que necesito, y alguna vez he necesitado, el apoyo de la empresa. Porque a veces te sientes que vas por libre, que eres tú la persona que..., porque, claro, el usuario sólo que te ve a ti, y claro, tú les dices “no, es que yo pertenezco a una empresa, y la empresa nos dice tal y está en ese lugar, y si usted tiene un problema ha de llamar a este teléfono que le responderá esta persona o esta otra, que son mis coordinadoras". Pero muchas veces no quieren llamar a otra persona. "No, porque si estás tú, y vienes cada día a casa, te digo las cosas a ti”, pero hay cosas que no son mi trabajo tampoco. (Teresa)

La negociación de las tareas en el domicilio implica procesos de observación y generación de confianza que, progresivamente, permiten la adaptación o la introducción de cambios. En este sentido, una TF ha de tener respeto y empatía y saber adaptarse a diferentes situaciones. Es importante respetar las costumbres y hábitos de la persona usuaria, a la vez que esta se tiene que adaptar a las normas del servicio. No se trata de imponer unas tareas en un domicilio sino de saber actuar dentro de unos marcos definidos por el servicio, para encontrar la comodidad de la TF y la satisfacción del usuario. Esto es lo que marca la relación profesional con ellos:

Hay de todo, entonces tienes que adaptarte a la clase de persona que es (...), tienes que ser un poco camaleón (...). Tienen maneras diferentes, niveles culturales diferentes. Con una persona eres de una manera y con otra eres de otra, dentro de tu profesionalidad. Has de ser un poco ambivalente con la gente. No todo el mundo necesita lo mismo ni todos lo ven del mismo color. (Ángeles)

No obstante, en su práctica profesional, aparecen tensiones que se resuelven mediante la negociación de la norma en cada caso, porque cada domicilio es un mundo y las TF se encuentran en procesos de negociación constantes. El apoyo de la empresa en este sentido les ayuda a delimitar las tareas y su práctica profesional. Al no existir un código deontológico de la profesión en España y sabiendo que los límites a veces son difíciles de negociar, la empresa establece unas normas de intervención en el domicilio elaboradas a partir de la práctica profesional. Aun así, hay normas difíciles de negociar y se deben ir adaptando a cada caso. Las TF dicen que "haces de 
más y de menos": tomar un café en el domicilio, hacer un encargo fuera de horario o visitas cuando no hay servicio, dar el teléfono al usuario, aceptar regalos, etc. Son situaciones que posteriormente podrían dificultar el trabajo. Este hecho muestra la relación personal que se establece en el servicio.

Tanto las personas usuarias como las trabajadoras consideran que el vínculo y la confianza es esencial para poder realizar el trabajo. Las TF perciben que establecen un vínculo laboral con las personas usuarias del servicio, quedando la empresa al margen de su rutina diaria. En este sentido, el trabajo de la TF está identificado con la intervención en el domicilio. Tal y como la mayoría de ellas expresan, su lugar de trabajo son los domicilios, no la empresa. Esta situación les genera contradicciones, porque van a trabajar a los domicilios, pero las condiciones las establece la empresa (representada principalmente por la figura de la coordinadora), con quien mantienen un contacto puntual. Este hecho provoca que tanto las TF como las personas usuarias sientan los casos como propios y no que formen parte de una organización, lo cual dificulta la gestión de reclamaciones por parte de las personas usuarias, o la propia organización del servicio, como sería el cambio de las profesionales en los domicilios debido a los requerimientos del trabajo (suplentes de las profesionales: vacaciones, bajas laborales, sobrecarga de las TF, etc.), cambios en los horarios del servicio, etc. La empresa en este contexto cumple la función de intermediaria entre los domicilios y los servicios sociales.

El trabajo en los domicilios se diferencia de otros trabajos de cuidado en instituciones (residencias u hospitales), donde las normas institucionales están muy presentes, se mecaniza más el trabajo, y se delimitan muy claramente las tareas en función de la categoría profesional. Por otra parte, el trabajo de cuidados en el domicilio se sigue asociando a lo privado, con poco reconocimiento y valor social. No pasa lo mismo, según el parecer de las TF entrevistadas, con el trabajo de cuidados en residencias, puesto que, al ser un trabajo desarrollado en una institución, se asocia al ámbito público y el reconocimiento es mayor, aparte de ser un trabajo mejor remunerado. Se mantiene, por tanto, la falsa dicotomía público/privado que otorga menos valor al ámbito privado, por ser un espacio de mujeres donde tradicionalmente se ha realizado un trabajo no reconocido e invisible (Himmelweit, 2000; Carrasco, 2009). Así pues, el servicio de cuidado realizado en los domicilios privados de las personas usuarias da continuidad al imaginario que establece la distinción público/privado, haciendo invisible el trabajo y perpetuando la idea del cuidado en la domesticidad. En este sentido, abordar el cuidado como una actividad profesional dentro del ámbito doméstico comporta un cambio de perspectiva en la relación laboral.

El SAD como recurso está bien valorado por las TF. Valoran positivamente que la persona usuaria pueda seguir viviendo en su domicilio y coinciden en que se ofrece una buena atención por el grado de personalización que se da. Pero el bienestar del usuario no se corresponde a veces con el bienestar de las profesionales y la calidad del cuidado. Las horas asignadas a cada usuario son muchas veces insuficientes para cubrir las necesidades de atención. Al ser un servicio puntual a unas necesidades más amplias, se delega buena parte del cuidado a los familiares. Esta idea es reforzada por las TF, que constatan que las personas usuarias creen que la responsabilidad del cuidado debe recaer en las familias, tal y como nos comenta Rosa: "por ejemplo, tienen hijos, y piensan que no debería venir una de fuera, una extraña". Así pues, la propia tipología del servicio se sustenta en la responsabilidad familiar del cuidado (Martínez-Buján, 2010). 
Hay situaciones donde se encuentran con dificultades para realizar su trabajo derivadas de las condiciones de precariedad y características de la vivienda (barreras arquitectónicas, falta de ayudas técnicas, etc.), que obstaculizan y ponen en riesgo a la persona usuaria y a ellas mismas (muchas de las bajas laborales son debidas a accidentes laborales). Tal y como comenta Susana, una TF: "Te encuentras con muchas dificultades en el momento de hacer la higiene y para movilizar a las personas porque, claro, los domicilios no están adaptados. La mayoría no están en condiciones". A pesar de informar de la situación, no se ponen medidas para mejorarla. Dicen que no se sienten apoyadas profesionalmente por la empresa ni por los servicios sociales, y cuentan con pocos recursos técnicos y emocionales para realizar el trabajo. En este sentido, es preciso reflexionar sobre el modelo de servicio y la calidad del mismo, teniendo en cuenta tanto el bienestar del usuario como de la profesional. A pesar de ello, la experiencia laboral anterior de muchas de ellas como cuidadoras profesionales en residencias les hace coincidir con que el SAD es el ámbito que más les gusta, porque les permite tener un trato más personalizado con las personas usuarias y les da cierto margen de iniciativa. Encuentran este trabajo más enriquecedor a nivel humano.

\subsection{La identidad profesional: Entre lo vocacional y lo profesional}

Lo que ha llevado a las TF a realizar este trabajo es que les gusta, se sienten útiles por la tarea que realizan. El hecho de ayudar a otra persona les satisface a nivel personal. Coinciden en el hecho de que es un trabajo que no lo puede realizar cualquier persona, pues se requieren unas cualidades, una disposición, una vocación y una implicación personal. Tal y como manifiesta Rosario "Lo llevo desde dentro, llevo toda la vida cuidando enfermos. Esta es una profesión que te tiene que salir a ti del corazón también". Todas coinciden, sin embargo, que su labor implica una vertiente profesional y una vertiente personal. Una TF ha de saber dónde comienzan y acaban sus funciones, tanto para limitar la exigencia de muchas personas usuarias como las propias ante situaciones emocionalmente complejas. Este aspecto es relevante en cuanto a la percepción que tienen las TF de esta profesión: entienden que realizan un trabajo porque están inmersas en una relación laboral, pero delimitar la actividad profesional es complejo porque también hay una parte personal. En función de la concepción que tienen del trabajo y de su grado de implicación personal, las TF consideran que la labor que realizan en el domicilio es un trabajo o no. Este aspecto, tal y como comenta Rosa, es significativo en cuanto a la percepción que tienen de un trabajo que tradicionalmente ha estado invisibilizado:

Te tienes que implicar mucho, y yo considero que este trabajo, o sea, esto no es un trabajo, ¿vale? Esto no es como vender una barra de pan o coser una camiseta, aquí si tú no pones sentimiento... Tú no vas al domicilio, fichas, hago y me voy..., tiene que haber un sentimiento hacia la persona y entregarte. Te tienes que entregar, no se puede ser fría. (Rosa)

A menudo, las TF no se sienten tratadas como profesionales por parte de las personas usuarias o sus familiares, ya que se prioriza su rol de cuidadoras, lo cual también dificulta la realización del trabajo y la delimitación profesional. María nos 
comenta que "para ellos es como algo familiar (...) es un servicio profesional pero a la vez es familiar". Esta identidad procede del entorno familiar, del papel de estas mujeres dentro de la familia, lo que se constata en la trayectoria de las trabajadoras entrevistadas, muchas de ellas cuidadoras familiares. El trabajo de cuidado a cargo de las TF, por el hecho de realizarse en el espacio doméstico, comporta también una confusión de roles, sobre todo para las mujeres, ya que su realidad vivencial es más compleja por el hecho de haber percibido la separación de los espacios (público/ privado) de forma impuesta, en oposición a los hombres, que tradicionalmente no se han hecho cargo del cuidado y han desarrollado su actividad en el espacio público (Carrasco, 2009). En este sentido, el trabajo familiar en el domicilio se sigue asociando a lo privado, lo que dificulta un reconocimiento de la labor profesional (Martin Palomo, 2008) y da continuidad al imaginario de cuidado familiar: la trabajadora deviene uno más de la familia.

Hay unas cualidades personales necesarias para realizar el cuidado, unas competencias que se asocian a las cualidades de la persona (a las mujeres) y que se adquieren con la socialización. Una $\mathrm{TF}$ ha de ser una persona cálida, empática y afectiva, debe de tener unas cualidades que tradicionalmente han sido feminizadas y tener un saber y una disposición sin las cuales los trabajos de cuidado no tienen sentido, tomando la idea de Torns (2008). Rosa, una de las TF lo expresa muy bien:

Es una persona que tiene que saber estar, y dar. Si no tienes paciencia, si no sabes comprender, dar cariño, escuchar..., no eres una TF. Una TF no es una persona que va ahí y hace una ducha, recoge y se va; hace una compra, una cama y se va... No..., eso es una parte de la TF... La TF se lleva dentro, es una personalidad, tienes que tener más funciones, tienes que saber escuchar, comprender, no tener en cuenta, ni reprocharle, ni encararte a esta persona... Tenemos que tener muchas cualidades, quien no las tenga yo creo que no debería estar en este trabajo. Yo lo habría dejado. (Rosa)

Son competencias adquiridas históricamente dentro de la familia y utilizadas por el mercado, que si bien son necesarias para el bienestar social, han permanecido invisibles (Carrasco, Borderías, Torns, 2011). Estas "cualidades femeninas", asociadas al género, responden a construcciones sociales vinculadas a valoraciones sociales y traducciones salariales. Responden a unas competencias que no han podido ajustarse a los criterios de valoración que derivan del mercado o de las políticas (Borderías, 2000). Se trata de unas cualidades reforzadas por las propias TF, por la empresa y por las personas usuarias del servicio. El jefe de servicio del SAD, una de las coordinadoras y algunas TF creen que hay una parte biológica en estas disposiciones, mientras que otras TF consideran que es una cuestión cultural. Aun así, todos coinciden en que las mujeres tienen una mejor disposición personal que los hombres para este trabajo. Esto es reforzado por las personas usuarias, que son reticentes a que sea un hombre quien las cuide, y por los servicios sociales y la empresa, que no promueven la contratación de hombres.

Supongo que somos más sensibles, ellos son más duros. (...) Yo he notado..., yo sé de muchos casos..., "a mí que no me venga un hombre", es que incluso a mí misma se me tapan, mujeres mismas se me tapan, y yo le gasto bromitas, "pero si estoy harta de verte cada día! Y si tuviera que venir un hombre, ¿qué?” "Ah, no, 
a mí un hombre no me toca! A mí un hombre no me ve!". Supongo que esto les frena más. (Rosa)

En general, las TF expresan que su trabajo también lo pueden desarrollar los hombres, pero ellas no se sienten identificadas con ellos, por lo que no reivindican que se contraten hombres sino que muestran una actitud permisiva en abrirles la profesión. La entrada de los hombres en la profesión, sin embargo, implica un cambio de mentalidad, sobre todo entre las usuarias del servicio, que son las más reticentes a los hombres cuidadores:

Yo pienso que no es lo mismo, la gente está acostumbrada a que las mujeres son quienes han de hacer esto. Claro, por ejemplo, un hombre, irle a hacer una limpieza a una mujer, y además, a una mujer mayor, como que no lo llevaría muy bien, ¿no? Con un hombre yo pienso que le sería más cómodo que le hiciera la limpieza, pienso. (María)

El único hombre que integra la plantilla de TF está contratado por la empresa desde 2010 y realiza las tareas de la profesión "menos femeninas", las que requieren más esfuerzo físico y un trabajo más educativo. Él mismo se inclina más por usuarios hombres, con problemas de salud mental o discapacidades. De las cualidades que se requieren para desarrollar las tareas, piensa que la comunicación es la más importante. Ahora bien, si entendemos que esta actitud frente a los demás es una característica de las mujeres, pero no por el hecho biológico sino por un determinado proceso de socialización, ¿los hombres también podrían adquirir esta disposición? Esta cuestión es objeto de debate y está recibiendo una respuesta positiva, ya que cada vez son más los hombres que asumen tareas del cuidado, conformando un nuevo deber en los comportamientos masculinos.

El trabajo de cuidado que realizan las TF en el domicilio implica una relación laboral pero también una relación emocional que se establece entre la profesional y el usuario. Es precisamente el aspecto relacional, el vínculo personal con la persona usuaria, lo que caracteriza este trabajo, que tal y como expresa Teresa condiciona el trabajo:

Con estos servicios que fluye esta empatía, que el usuario está..., que está agradecido contigo, que vengas aquí, que le hagas esto, que le hagas aquello... La relación es formidable, hay una empatía, hay un cariño. Que si esta persona está bien, tú te contentas de que está bien, que si está enferma, te sientes mal porque está enferma. Pero claro, en los servicios que no hay esto, es diferente. (Teresa)

Las TF valoran las habilidades personales para realizar su trabajo, pero refuerzan también las capacidades técnicas que se adquieren principalmente mediante la formación y también con la práctica profesional. Así, se va configurando un perfil profesional integrado por un saber humano y un saber técnico, que nos lleva a reflexionar sobre la complejidad del cuidado, el cual engloba tanto aspectos materiales como emocionales (Hochschild, 2008; Molinier, 2008; Zelizer, 2009). En este sentido el cuidado implica un vínculo afectivo entre quien brinda y quien recibe, está basado en lo relacional, por lo cual también involucra emociones que se expresan en las relaciones, ya sean familiares o remuneradas (Hochschild, 2008; Zelizer, 2009). En 
el día a día, las TF se encuentran con variedad de situaciones y problemáticas en los domicilios (temas concretos de salud mental, patologías diversas, etc.), por eso creen que la formación especializada es importante. Reciben poca formación por parte de la empresa y valoran que ésta les ofrezca cursos para ampliar sus conocimientos y mejorar la atención. Consideran, no obstante, que las personas usuarias (y la sociedad) desconocen que se requieren unos conocimientos técnicos para desarrollar su trabajo, con lo que se da continuidad al imaginario de que cualquier mujer puede ser cuidadora:

No miran la importancia de tener los conocimientos de ver cuándo a una persona le está dando una bajada de azúcar. Tienes que estar preparada para todo, pues te puedes poner a fregar los platos, como tienes que estar atenta a que esta persona se esté poniendo más blanca, esta persona se está mareando. Tienes que estar preparada. (...) estar más preparado, tanto con estudios y con prácticas. Porque la mitad de la gente yo pienso que no saben cómo coger a un enfermo, y ¿qué pasa? Que se hacen daño... Hacer un baño, lo mismo. Tú no puedes empezar por el culete y acabar con la cara, lo mismo con una cura, cambiar pañales. (María)

Muchas de ellas comenzaron sin formación, con la experiencia de tener que cuidar a un familiar. Otras también disponían de experiencia personal como cuidadoras y consideraban la formación como una oportunidad para dar continuidad al trabajo que realizaban (como cuidadoras familiares o en domicilios privados), pero de forma reglada, para así poder optar a trabajos cualificados y entrar en el mercado remunerado formal. La socialización de clase de estas mujeres y la dificultad de acceder a empleos de más prestigio y mejor pagados, refleja la desigualdad social en este trabajo. Aunque desde 2003 se ha reconocido la profesión con la titulación de Técnico de Atención Sociosanitaria, responde a una cualificación baja. Por otra parte, con la acreditación de competencias ${ }^{11}$, la experiencia laboral aparece como una alternativa legítima a la cualificación. Este proceso que refuerza los saberes femeninos y descansa en la naturalización y feminización del trabajo de cuidado, tampoco ayuda a la profesionalización del sector.

\subsection{El contenido del trabajo de cuidados}

Las tareas a realizar en el SAD que se describen en los pliegos técnicos del Ayuntamiento y en el convenio laboral del sector incluyen la parte material de las mismas (control de la medicación, transferencias dentro del hogar, preparar la comida, etc.), pero las TF describen la actividad que realizan en el domicilio tanto material como inmaterial. En este sentido la actividad de cuidado engloba tanto el aspecto técnico como emocional. La disposición y la actitud hacia la persona que se atiende:

Yo tengo casos en los que es movilización continua, pero dentro de esta movilización está el emocional, el personal, y todo (...) Dentro de esta movilización tengo un contacto directo con esta persona, si tengo que gastarle una broma para animarle, se la gasto. (Rosa)

11 En Cataluña corresponde a los programas acredita 't o qualifica't, que posibilita un reconocimiento de las competencias profesionales mediante la experiencia laboral y formación no reglada. 
Sin embargo, esta naturaleza del cuidado comporta unos valores relacionales que entran en contradicción con los valores de producción de la empresa (Pérez Orozco, 2006). Mientras que el cuidado está configurado por dos dimensiones (material y afectiva), los bienes mercantiles o públicos contemplan la dimensión más objetiva de las necesidades, de forma que mecanizan el servicio. Las trabajadoras, aun así, incluyen el afecto y las relaciones implícitas en la necesidad objetiva que se satisface, y durante su servicio, por más que estén en unas condiciones mercantiles, existen relaciones de afecto. Poner límites de un trabajo emocional realizado en un espacio íntimo y personal en el marco de unas relaciones laborales es necesario para delimitar el trabajo: "O sea, una atención continua siempre para lo que necesitan, pero, a ver, un límite afectivo lo has de poner. Porque no es bueno ni para ellos ni para ti..., estás trabajando..., con personas, pero es un trabajo, no es tu familia" (Rosa). en esta dirección, reclaman más coordinación y seguimiento, mejores condiciones y un apoyo psicológico por parte de la organización.

En el SAD se especifican qué tareas se tienen que realizar, subdivididas en dos categorías: limpieza del hogar (relacionada en el mantenimiento material de la vida y en las condiciones de los espacios de la reproducción social) y atención a la persona (relacionada con la reproducción de la vida y el bienestar). Las primeras las desarrollan las Auxiliares del Hogar (AH) y las segundas, las TF. Se establece entonces una diferenciación de las actividades en función de su prestigio social. Se trata de diferenciaciones simbólicas, que se reflejan también en la categoría social de las profesionales, teniendo las $\mathrm{AH}$ menos cualificación y una remuneración más baja $^{12}$. A pesar de esta diferenciación normativa, a menudo, la delimitación de tareas suscita tensiones, dado que las actividades no quedan bien delimitadas. Para las TF, al concebir las actividades a desarrollar en el domicilio como "domésticas", lo que las mujeres realizan tradicionalmente en el ámbito doméstico, muchas veces acaban asumiendo la totalidad de las tareas (incluyendo la limpieza del hogar). Esta diferenciación es confusa también para las personas usuarias y las TF, de manera que las actividades no quedan bien delimitadas. Las TF son consideradas una "chacha para todo", alguien que cuida y que, además, asume la limpieza de la casa: "Yo, cuando llegaba, me decía "limpia esto, limpia lo otro", y yo le decía "es que yo no..., ya viene una mujer de la limpieza, yo no vengo a quitar el polvo". Conscientes de esta ambigüedad, las TF se diferencian de las AH, circunscribiendo su trabajo a las tareas que les son propias. Creen que es importante que las personas usuarias (la empresa y los servicios sociales) sepan diferenciar quiénes son y qué hacen, de esta manera se sienten más valoradas que las AH, dan sentido a su trabajo, refuerzan su identidad como grupo y contribuyen al sentimiento de profesionalidad, al mismo tiempo que obtienen un reconocimiento:

Trabajadora familiar la veo un poco ambigua, que un poco significa una cosa, un poco significa otra, o sea, depende del punto de vista que tengas, porque muchas veces dicen “¿qué es eso de trabajadora familiar?”. Se conoce poco, y se tiende a

12 El IV Convenio colectivo de empresas de atención domiciliaria de Cataluña establece las dos categorías (auxiliar de limpieza y trabajadora familiar o auxiliar de geriatría), con distintas remuneraciones. Sin embargo, el VI Convenio Marc Estatal de servicios de atención a las personas dependientes y desarrollo de la autonomía personal establece una única categoría laboral (Auxiliar de Ayuda a Domicilio) para realizar todas las tareas domésticas. 
confundir. “Ah, ¿tú eres la mujer..., qué eres? ¿La que vas a limpiar, la que vas a hacer la compra? (Teresa)

El cuidado se vincula a las tareas que tradicionalmente las mujeres han desarrollado en el ámbito doméstico, poco valoradas e invisibilidadas, y esto contribuye a que el trabajo remunerado de cuidados se considere una extensión del trabajo doméstico. Teniendo en cuenta que el cuidado engloba tanto las tareas de atención como domésticas, esta diferenciación es producto de la organización del servicio y de la clasificación profesional. Este aspecto también responde al hecho de que el traslado del trabajo doméstico a la esfera remunerada siga manteniendo las mismas características, ya que el cuidado abarca tanto el cuidado directo a las personas como el indirecto, es decir, el trabajo doméstico que es un requisito previo para que el cuidado directo pueda prestarse (Razavi, 2007, Esquivel, 2011).

\section{Conclusiones}

En este artículo hemos analizado el modelo de cuidado a personas mayores ofertado desde el Servicio de Atención a Domicilio (SAD). Un trabajo que convive con formas tradicionales de atención a cargo de la familia, principalmente por parte de las mujeres y donde la profesionalización no está consolidada. El SAD es un escenario que refleja las dificultades del traslado del cuidado de los entornos íntimos a la esfera pública o remunerada y las desigualdades de clase, género y etnia, siendo las trabajadoras del SAD mujeres, principalmente autóctonas, con poca formación, que trabajan en condiciones precarias.

Durante la práctica profesional del cuidado, aparecen tensiones y ambivalencias que vienen dadas por distintos factores: la organización del servicio, el carácter doméstico de las actividades, porque se trata de una actividad feminizada (son consideradas cuidadoras naturales), el lugar donde se realiza el cuidado (espacio privado), así como el modelo de política (privatizadora). El Trabajo Familiar es una profesión feminizada, muchas de las TF tienen experiencia como cuidadoras familiares y se identifican con este trabajo por ser mujeres. En este sentido, la identidad de las TF entrevistadas comporta una confusión de roles y dificulta el reconocimiento social de la profesión. Por otra parte, el hecho de que las TF sean mayoritariamente mujeres que realizan su labor en los domicilios genera tensiones que es necesario negociar para delimitar la actividad como profesión y para dar valor a este tipo de trabajo. El lugar de trabajo tampoco contribuye al sentimiento de pertenencia a una organización (trabajadoras de una empresa). Las trabajadoras entran en conflicto con el funcionamiento de la empresa porque las ajustadas condiciones laborales y los insuficientes recursos condicionan la calidad del servicio. No se tienen en cuenta las dimensiones del trabajo emocional que existen a pesar de ser un trabajo bajo condiciones mercantiles. Sin embargo, ellas se implican en el trabajo, tanto personal como profesionalmente. No es extraña la concepción ambivalente que las TF y las personas usuarias tienen de su trabajo si entendemos que la sociedad se ha organizado según la dicotomía de esfera reproductiva (no remunerada y femenina) y esfera productiva (remunerada y masculina). Estas ambivalencias conllevan dificultades en la realización del cuidado remunerado y en la delimitación del trabajo de cuidado como profesión y plantean la necesidad 
de nuevos debates sobre la responsabilidad social del cuidado y las características propias de la actividad cuando ésta es remunerada.

La estrategia política de "desfamiliarización", es decir, el traspaso de la responsabilidad de cuidado del ámbito privado al público, no aborda el problema de las desigualdades de género y clase, siendo la mujer la que continúa realizando las tareas de cuidado, tanto en el ámbito remunerado como no remunerado. Con la creación de estos servicios, si bien se da la oportunidad de dar reconocimiento a estos "saberes femeninos", perdura aún su vinculación al ámbito doméstico, el no reconocimiento y la escasa valoración social, que, como hemos visto, se refleja en las condiciones precarias y los bajos salarios de esta profesión. De acuerdo con lo analizado, muchas de estas mujeres tienen poca formación y encuentran en este ámbito una posibilidad de inserción laboral. Son empleadas en el SAD dando continuidad a su trayectoria personal como cuidadoras, debido tanto a la socialización de clase como a la dificultad de acceder a empleos de más prestigio y mejor pagados. El hecho que la mayoría de las TF sean autóctonas muestra una distribución diferencial de las trabajadoras de cuidados en distintos empleos según su origen, siendo el empleo doméstico protagonizado por mujeres inmigradas (muchas veces sin formación), además de ser menos valorado y regulado que el profesional. Además el SAD si bien posibilita la permanencia de la persona en el domicilio, se sustenta en la responsabilidad individual y familiar del cuidado y da continuidad a la desigual distribución de los trabajos domésticos. En este sentido, el traslado de la actividad de cuidados a la esfera remunerada, en el marco del SAD, necesita ser redefinida: tanto la organización, la provisión y el contenido del trabajo como las imágenes culturales entorno al cuidado.

\section{Bibliografía}

Asociación Catalana de Recursos Asistenciales (2014). Informe econòmic i social ACRA del sector d'atenció a les persones grans amb dependència a Catalunya. (en línea)

http://itemsweb.esade.edu/idgp/Presentacio_2014_InformeEconomicACRA.pdf.

Batthyány, K., Genta, N. y Perrotta, V. (2014). "Las representaciones sociales del cuidado infantil desde una perspectiva de género. Principales resultados de la encuesta nacional sobre representaciones sociales del cuidado". Papers. 99 (3): 335-354.

Borderías, C. (2000). "La feminización de los estudios sobre el trabajo de las mujeres (19691999)". En: Heredero, C. (coord.). Mujeres: unidad y diversidad. Un debate sobre la identidad de género (pp. 51-93). Madrid: Federación de enseñanza de CC.OO.

Brullet, C. (2010). "Cambios familiares y nuevas políticas sociales en España y Cataluña. El cuidado de la vida cotidiana a lo largo del ciclo de vida". Educar. 45: 51-79.

Butler, J. (1990). El género en disputa. Barcelona: Paidós.

Carrasco, C., Borderías, C. y Torns, T. (2011). "Introducción. El trabajo de cuidados: antecedentes históricos y debates actuales". En Carrasco, C; Borderías, C y Torns, T. El trabajo de cuidados. Historia, teoría y políticas (pp.13-95). Madrid: La catarata.

Carrasco, C. (2009). "Mujeres, sostenibilidad y deuda social". Revista de educación. número extraordinario: 169-191.

Comas, D y Roca, J. (1996). "El cuidado y asistencia como ámbito de expresión de la tensión entre biología y cultura". En Contreras, J. (coord). Reciprocidad, cooperación y organización comunal. VIII Congreso de Antropología social, Zaragoza 
Comas d'Argemir, D. (2012). "Políticas públicas y vida cotidiana. Redescubrimiento y auge de las políticas familiares en España”. Scripta nova. Revista electrónica de geografía y ciencias sociales. vol. XVI, 395 (20). (en línea) http://www.ub.es/geocrit/sn/sn-395/sn395-20.htm.

Comas d'Argemir, D. (2015). "Los cuidados de larga duración y el cuarto pilar del sistema de bienestar". Revista Antropología Social. 24: 375-404.

Daly, M. y Lewis, J. (2000). "The concept of social care and the analysis of contemporary welfare states". The british journal of sociology. 51(2): 281-298.

Del Valle, T. (2004). "Contenidos y significados de nuevas formas de cuidado", en Actas Congreso Internacional sare 2003: Cuidar cuesta: costes y beneficios del cuidado, Vitoria: Ekamunde.

Deusdad, B., Comas d'Argemir, D. y Dziegielewski, S. (2016). "Reestructuring long term care in Spain: The Impact of The Economic Crisis on Social Policies and Social Work Practice". Journal of Social Service Research. 42(2): 246-262.

Duran, M. A. (2014). "Mujeres y hombres ante la situación de dependencia", en Seminario: Políticas públicas de atención a personas mayores dependientes: Hacia un sistema integral de cuidados, Madrid: Instituto de Mayores y Servicios Sociales.

Esquivel, V. (2011). La economía del cuidado en américa latina: poniendo a los cuidados en el centro de la agenda. Programa Naciones Unidas para el Desarrollo: Panamá.

Fraser, N. (1997). Reflexiones críticas desde la posición "postsocialista”. Santafé de Bogotá: Universidad de los Andes/Siglo del Hombre Editores.

Himmelweit. S. (Ed.) (2000). Inside the household: From Labour to Care. Hereford: Palgrave Macmillan.

Hochschild, A.R. (2008). La mercantilización de la vida íntima. Madrid: katz.

Instituto de Mayores y Servicios Sociales. (2004). Libro Blanco de atención a las personas en situación de dependencia. (en línea) http://www.dependencia.imserso.es/InterPresent1/ groups/imserso/.../libroblanco.pdf.

Instituto de Mayores y Servicios Sociales (2012). Información estadística del Sistema para la autonomía y atención a la dependencia. (en línea)

http://www.dependencia.imserso.gob.es/InterPresent2/groups/imserso/documents/binario/ im_070847.pdf

Juliano, M.D. (1989). “Estrategias de elaboración de la identidad”. Realitat. 13: 9-23.

Lewis, J. (2007). "Género, envejecimiento y el nuevo pacto social: la importancia de desarrollar un enfoque holístico de las políticas de cuidados”. En Carrasco, C; Borderías, C y Torns, T. El trabajo de cuidados. Historia, teoría y políticas (pp.336-358). Madrid: La catarata.

Mandell, B.R. (Ed.). (2010). The crisis of caregiving. Social welfare policy in the united states. New York: Palgrave Macmillan.

Marbán Gallego, V. (2011). "Los actores sociales en el sistema de dependencia español”. Cuadernos de Relaciones Laborales. 29(1): 69-91.

Martin Palomo, M. T. (2008). “Domesticar” el trabajo: una reflexión a partir de los cuidados. Cuadernos de Relaciones Laborales. 26(2): 13-44.

Martínez-Buján, R. (2010). Bienestar y cuidados: el oficio del cariño. Mujeres inmigrantes y mayores nativos. Madrid: Consejo superior de investigaciones científicas.

Martínez-Buján, R. (2011). La reorganización de los cuidados familiares en un contexto de migración internacional. Cuadernos de Relaciones Laborales, 29(1): 93-123.

Molinier, P. (2008). Trabajo y compasión en el mundo hospitalario. Una aproximación a través de la psicodinámica del trabajo. Cuadernos de Relaciones Laborales. 26:121-138. 
OECD/European Commission. (2013). A Good Life in Old Age? Monitoring and Improving Quality in Long-term Care. Paris: OECD. (en línea) http://dx.doi.org/10.1787/9789264194564-en

Otaegui, A. (2007). "Presentación". En Escudero, R, La calidad del Empleo del nuevo sistema para la autonomía personal y atención a la dependencia (pp. 21-33). Alzira: Germania.

Parella, S. (2003). Mujer, inmigrante y trabajadora: la triple discriminación. Barcelona: Anthropos.

Pérez Orozco, A. (2006). "Amenaza tormenta: la crisis de los cuidados y la reorganización del sistema económico". Revista de economía crítica. 5: 7-37.

Peterson, E. (2015). Framing caregiving work for older people in Spanish public policy: gender, power and social justice. Revista Española de Ciencia Política. 39: 221-237.

Recio, C. (2010). "Familismo, asistencialismo y precariedad. La configuración del empleo en el sector de atención a las personas". Alternativas. 17: 19-43.

Recio, C., Moreno, S., Borràs, V. y Torns, T. (2015). "La profesionalización de sector de los cuidados". Zerbituan. 60: 179-193.

Razavi, S. (2007). The Political and Social Economy of Care in a Development Context. Conceptual issues, research questions and policy options. Ginebra: UNRISD Programme Paper.

Razavi, S. y Staab, S. (2010). "Mucho trabajo y poco salario. Perspectiva internacional de los trabajadores del cuidado". Revista internacional del trabajo. 29 (4): 449-467.

Rodríguez Cabrero, G. (2011). "Políticas sociales de atención a la Dependencia en los regímenes de bienestar de la unión europea". Cuadernos de Relaciones Laborales. 291:13-42.

Rogero-García, J. y Ahmed-Mohamed, K. (2014). "What is the best care for community-dwelling dependent adults? Sources of care and perception of unmet needs in Spain". Revista Internacional de Sociología. 72(2): 403-427.

Stolcke, V. (2003). "La mujer es puro cuento: la cultura del género". Quaderns de l'Institut Català d'Antropologia, serie monogràfics: A propòsit de la cultura. 19: 69-95.

Tobío, C., Agulló T., Gómez, M. V. y Martín Palomo, M. T. (2010). El cuidado de las personas. Un reto para el siglo XXI. Barcelona: Fundació "la Caixa".

Torns, T. (2008). "Les polítiques per a l'organització social de la cura a l'esfera local. Implicacions des de la perspectiva de gènere". En De la Fuente Vàzquez, M. (coord.) Les àrees de serveis personals: donant perspectiva de gènere a les polítiques socials d'àmbit local. Barcelona: Institut de ciències polítiques i socials.

Tronto, J. (2005). "Cuando la ciudadanía se cuida: una paradoja neoliberal del Bienestar y la desigualdad" en Actas Congreso Internacional Sare 2004: Hacia que modelo de ciudadanía, Vitoria: Ekamunde.

Vega, C. (2009). Culturas del cuidado en transición. Espacios, sujetos e imaginarios en una sociedad de migración. Barcelona: Editorial UOC.

Vilà, A. (2013). "Evolución y reforma del sistema para la autonomía y atención a la dependencia", en Actas de la Dependencia Fundación Caser. 8: 49-69.

Waerness, K. (1987). "Sobre la racionalidad del cuidado". En: Sassoon, A.S. (ed.). Las mujeres y el estado. Madrid: Vindicación Feminista.

Williams, F. (2010). Claiming and framing in the making of care policies : The recognition and redistribution of care. Ginebra: UNRISD Programme Paper.

Zelizer, V. (2009). La negociación de la intimidad. Argentina: Fondo cultura económica. 